\title{
Le bilinguisme des enfants d'origine turque scolarisés en maternelle en France (en Alsace)
}

\author{
Hamurcu Süverdem, Büşra \& Akinci, Mehmet-Ali \\ Laboratoire DyLiS, Université de Rouen \\ busrahamurcu@gmail.com et mehmet-ali.akinci@univ-rouen.fr
}

\begin{abstract}
Résumé. Le bilinguisme des enfants issus de familles immigrées a inspiré beaucoup de recherches. Or, peu d'entre elles s'intéressent au développement des compétences interactionnelles de ces bilingues émergents (Garcia, 2009) dans leurs deux langues et au rôle des pratiques langagières familiales de ces enfants dans leur développement langagier bilingue. Il s'agira, donc, dans cette étude, d'observer les compétences interactionnelles de six enfants bilingues franco-turcs entre 3 et 5 ans, ayant différentes pratiques langagières familiales, à savoir la pratique du turc uniquement pour certains et celle du turc et du français pour d'autres. Les compétences analysées sont la thématique, les genres (séquences discursives et sous-genres discursifs), les modalités d'énoncé et la diversité lexicale. Les résultats indiquent d'une part, un effet des différentes pratiques langagières familiales sur le développement langagier, et, d'autre part un effet de la scolarisation qui renforce la pratique du français.
\end{abstract}

\begin{abstract}
Many researchers have studied the bilingualism of Turkish children from immigrant background. However, very few studies focus on the development of interactional competences of these emergent bilinguals (Garcia, 2009) in both languages and on the role of language practices at home in their language developement. This study observes the development of interactional competences of six Turkish-French bilingual children children with an immigrant background since the first year until the end of the second year of preschool (3-5 years). These children are exposed to different language practices at home such as essentially Turkish (Type 1) and a mix of Turkish and French (Type 2). The corpus of data consists on verbal interactions between the adult and children. We have studied the thematic patterns in our interactions, the diversity of genres, the modalities of utterances and lexical diversity. The results show both that home language practices and schooling have an impact on the development of language competences.
\end{abstract}




\section{Introduction}

Le bilinguisme émergent (Garcia, 2009) des enfants issus de familles immigrées est souvent ignoré à l'école (Hélot, 2007), car d'une part, les enseignants ne sont pas attentifs au répertoire plurilingue de leurs élèves et d'autre part, ce sont parfois les enfants eux-mêmes qui ne manifestent pas leur bilinguisme (Akinci, à paraître 2016). Par conséquent, ces enfants se trouvent en insécurité linguistique à la fois parce qu'ils ne retrouvent plus leur L1 à l'école et aussi parce que leurs compétences, modestes, certes, mais existantes en L2 ne sont pas assez valorisées, ce qui crée chez les enfants un sentiment d'insuffisance dès les premiers jours de l'école. Ces enfants bilingues dont aucune des langues de leur répertoire n'est jugée « valable » à l'école se retrouvent très souvent dans le silence. Ce silence inquiète les enseignants qui ne trouvent parfois d'autre solution que de solliciter la pratique de la L2, la langue de l'école pour que l'enfant puisse rapidement « entrer dans les apprentissages » comme ils le disent souvent.

Les travaux en acquisition du langage avant l'âge scolaire et pendant la période de l'école maternelle, que ce soit en contexte monolingue ou bi/plurilingue s'intéressent en général aux compétences purement linguistiques des enfants en étudiant des corpus de productions orales. Or, dans une approche interactionniste, (Vygotsky, 1934 ; Bruner, 1983 ; Kerbrat-Orecchionni, 1990 ; François, 1990) il est davantage intéressant d'observer l'intégralité des interactions dans lesquelles s'inscrivent ces productions orales afin de pouvoir analyser, de manière plus générale, les compétences interactionnelles des enfants. En effet, si nous souhaitons analyser le développement des capacités langagières des jeunes bilingues, il n'est pas pertinent de se limiter au développement des capacités purement linguistiques car elles ne représentent pas la totalité de tout ce dont l'enfant est capable au niveau langagier.

Cet article présente et discute les résultats des analyses interactionnelles et linguistiques recueillis auprès de six enfants bilingues franco-turcs scolarisés en maternelle (Hamurcu Süverdem, 2015). Ces analyses ont été menées sous quatre volets : l'analyse de la thématique, l'analyse discursive, à savoir l'analyse des types de séquences discursives et des sous-genres discursifs, l'analyse des modalités d'énoncé et l'analyse de la diversité lexicale dans les interactions. Dans un premier temps, nous dresseronsun bref historique de l'immigration turque en France pour mieux présenter nos informateurs par la suite. Dans un second temps, nous présenterons nos informateurs et notre méthodologie de recueil de données. Enfin, dans un dernier temps, nous exposerons nos résultats.

\section{Immigration turque : $\mathbf{5 0}$ ans de présence en France}

D'après l'INSEE (2015), un immigré est « une personne née étrangère à l'étranger et résidant en France ». Cette définition n'inclut donc pas toutes les personnes d'origine turque vivant actuellement en France. En effet, elle décrit en majorité les caractéristiques des membres de la première génération immigrée pour des raisons économiques suite à la signature de la convention de la main-d'œuvre entre la France et la Turquie le 8 avril 1965. Elle inclut donc également les conjoint(e)s de ces travailleurs immigrés qui viennent en France grâce au regroupement familial à partir des années 1980. Les enfants de ces couples seront, 
pour la plupart, des étrangers nés en France et constitueront la deuxième génération qui sera plutôt désignée comme « issue de l'immigration » et non pas immigrée, car elle n'est pas directement acteur de l'immigration. Quant à la notion d' « étranger », c'est ainsi qu'elle est définie par l'INSEE (ibid.) :

\begin{abstract}
«Un étranger est une personne qui réside en France et ne possède pas la nationalité française [...]. Un étranger n'est pas forcément immigré, il peut être né en France (les mineurs notamment). A la différence de celle d'immigré, la qualité d'étranger ne perdure pas toujours tout au long de la vie : on peut, sous réserve que la législation en vigueur le permette, devenir français par acquisition. ».
\end{abstract}

Les membres de la deuxième génération ayant pu acquérir la nationalité française ne seront donc plus considérés comme «étrangers", mais des «Français issus de familles immigrées originaires de Turquie ». À partir des années 1990, le mariage des membres de cette deuxième génération avec des jeunes du pays d'origine sera à l'origine d'un nouveau flux migratoire : l'arrivée des gendres et des brus. Ces derniers sont également conformes à la définition de la notion d' "immigré » que nous avons citée plus haut. Parmi ces gendres et brus, ceux qui parviennent à obtenir la nationalité française ne seront plus «étrangers », mais resteront «immigrés », car sa définition précise également que même si certains immigrés ont l'opportunité de devenir français - alors que d'autres restent étrangers - «la qualité d'immigré est permanente : un individu continue à appartenir à la population immigrée même s'il devient français par acquisition ».

Les enfants nés de ces couples endogames (dans lequel les deux membres sont issus de la même culture et de la même communauté, à savoir la communauté turque en France) ou exogames (dans lequel chacun des membres sont issus de communautés différentes, à savoir l'un né et ayant grandi en France et l'autre en Turquie) constituent la troisième génération. Le cas des membres de cette dernière génération est tout autre, car d'une part, ils ne sont plus considérés comme "étrangers », étant donné qu'ils naissent en France, de parents français pour la plupart, et, d'autre part, ils ont le rapport le plus lointain avec le passé migratoire de leur famille, car soit, ils n'ont aucun parent, soit qu'un seul qui soit directement acteur de l'immigration. Les enfants appartenant à cette troisième génération seront eux aussi considérés comme des enfants «issus de familles immigrées » dans notre étude, mais précisons que cette appellation fait référence à la famille élargie et non pas la famille immédiate qui n'est pas toujours directement acteur de l'immigration comme nous l'avons expliqué plus haut. Cette précision est importante, car nos informateurs sont des membres de cette troisième génération dont nous venons de présenter les caractéristiques. Au point suivant, nous allons présenter nos informateurs de manière détaillée ainsi que notre méthodologie de collecte de données.

\title{
3 Les informateurs et la collecte de données
}

Nous avons choisi comme terrain de recherche une commune alsacienne fortement peuplée de Turcs qui y constituent $27 \%$ de la population. Cette commune était un terrain propice à la fois en raison de sa forte population turque et notre bonne connaissance d'une grande partie de cette population. Notre position de chercheur endogroupale nous a permis de construire rapidement une relation de confiance avec les familles et surtout les enfants qui sont nos informateurs. Nous avons sélectionné ces derniers dans les deux écoles maternelles de la commune les plus fréquentées par les enfants turcs. Ensuite, nous avons constitué deux groupes d'enfants que nous avons nommés Type 1 et Type 2, en fonction de leur pratiques langagières familiales, à savoir, respectivement, la pratique du turc uniquement et la pratique des deux langues (turc et français). Ainsi, nous avons suivi 6 enfants d'origine turque du début de la petite section de maternelle jusqu'à la fin de la moyenne section, à raison de les enregistrer individuellement 5 fois (3 en petite section et 2 en moyenne section) en turc dans un premier temps et en français dans un second temps, souvent avec une semaine d'intervalle. Certaines informations importantes à propos de ces derniers figurent dans le tableau ci-dessous : 
Tableau 1 : L'âge des enfants Type 1 et Type 2 et la moyenne d'âge à la première et à la dernière séance.

\begin{tabular}{|c|c|c|c|c|c|c|c|}
\hline \multicolumn{2}{|c|}{ Type } & \multicolumn{3}{|c|}{ Type 1 } & \multicolumn{3}{c|}{ Type 2 } \\
\hline \multicolumn{2}{|c|}{ Nom } & Ismail & Asli & Nur & Yelda & Isa & Yusuf \\
\hline \multicolumn{2}{|c|}{ Garçon/Fille } & G & F & F & F & G & G \\
\hline \multirow{4}{*}{$\begin{array}{c}\text { Âge } \\
\text { S1 (10/11) }\end{array}$} & $3 ; 7$ & $2 ; 10$ & $3 ; 5$ & $2 ; 10$ & $3 ; 8$ & $3 ; 2$ \\
\cline { 2 - 9 } (début PSM) & Moyenne d'âge & \multicolumn{3}{|c|}{$\mathbf{3 ; 3}$} & \multicolumn{4}{|c|}{$\mathbf{3 ; 2}$} \\
\cline { 2 - 9 } & S5 (06/13) & $5 ; 3$ & $4 ; 6$ & $5 ; 1$ & $4 ; 6$ & $5 ; 4$ & $4 ; 10$ \\
\cline { 2 - 8 } & Moyenne d'âge & \multicolumn{3}{|c|}{$\mathbf{4 ; 1 1}$} & \multicolumn{4}{|c|}{$\mathbf{4 ; 1 0}$} \\
\hline
\end{tabular}

Lors des séances d'enregistrement, nous nous sommes servi d'un support imagé (Kern \& Raffara, 2012) représentant des enfants qui jouent dans une cour d'école. Les interactions en turc et en français avec l'adulte ont été transcrites et analysées sous quatre volets d'analyse :

- la thématique qui étudie la distribution des propositions de thème de l'adulte et des enfants dans les interactions ;

- les genres qui se divisent en 2 sous-parties et qui étudient respectivement la nature des séquences discursives et celle des énoncés ;

- la modalité des énoncés qui en étudie la fonction ;

- $\quad$ et enfin la diversité lexicale des enfants dans les deux langues.

Nous allons détailler la présentation de ces catégories d'analyse et en présenter les résultats au point suivant.

\section{Les résultats}

\subsection{La thématique}

Nous savons qu'une interaction se divise toujours en plusieurs thèmes et sous-thèmes, car les interlocuteurs abordent plus d'un sujet lorsqu'ils sont en interaction. Dans ce premier volet d'analyse, nous n'avons pas fait l'inventaire de tous les thèmes abordés par les interlocuteurs, mais nous avons observé leur distribution en quantité en fonction de la langue de l'interaction, du type d'enfant et du locuteur qui introduit les thèmes. Le tableau ci-dessous présente le taux de proposition de thème pour chaque interlocuteur (adulte et enfants) en fonction de la langue de l'interaction. Ces résultats sont les moyennes de toutes les séances. 
Tableau 2: Le taux (en pourcentage) de proposition de thème chez les enfants Type 1 et Type 2 et chez l'adulte dans les interactions en turc et en français.

\begin{tabular}{|c|c|c|}
\hline Enfants Type 1 & Turc & Français \\
\hline Adulte & $52 \%$ & $73,7 \%$ \\
\hline Enfants & $48 \%$ & $26,3 \%$ \\
\hline
\end{tabular}

\begin{tabular}{|c|c|c|}
\hline Enfants Type 2 & Turc & Français \\
\hline Adulte & $65 \%$ & $60,5 \%$ \\
\hline Enfants & $35 \%$ & $39,5 \%$ \\
\hline
\end{tabular}

Les résultats ci-dessus montrent que c'est l'adulte qui domine les interactions en proposant plus de thèmes que les enfants quelle que soit la langue ou le type d'enfant. Seuls les enfants Type 1 proposent presque le même pourcentage de thème que l'adulte en turc. Nous pouvons donc en déduire que les interactions en turc avec les enfants Type 1 sont moins dominées par l'adulte.

En ce qui concerne la comparaison entre les deux types d'enfant, les enfants Type 1 proposent plus de thèmes en turc que les enfants Type $2(+13 \%)$. En revanche, en français, ce sont les enfants Type 2 qui proposent plus de thèmes que les enfants Type $1(+13,2 \%)$ dans la mesure où ils ont une pratique plus précoce du français.

La proposition de thème est donc intimement lié à l'aisance dans la langue de l'interaction, car, d'après les résultats, les enfants proposent plus de thèmes dans la langue où ils sont à l'aise et lorsqu'ils ne peuvent pas en proposer en raison des contraintes linguistiques, c'est l'adulte qui s'en charge.

\subsection{Les genres}

D’après Bakhtine (1984 : 285), la question du genre est inévitable en acquisition du langage. Il écrit :

\footnotetext{
«Apprendre à parler, c'est apprendre à structurer des énoncés (parce que nous parlons par énoncés et non par propositions isolées et, encore moins, bien entendu, par mots isolés). Les genres de discours organisent notre parole de la même façon que l'organisent les formes grammaticales (syntaxiques) ».
}

François (1993) complète en affirmant que les genres correspondent à ce que nous faisons avec le langage, à savoir décrire, raconter, expliquer, etc.Les genres sont souvent très hétérogènes, mélangés (François, 1993) et perméables (François \& Delamotte-Legrand, 2004). En effet, un genre peut faire appel à des sous-genres, ce qui est très souvent le cas dans notre corpus, comme dans tous discours. Or, tous les genres ne peuvent constituer des « organisateurs dominants » (François, 1993). Nous observons donc plus de sous-genres différents que de genres différents en tant qu'organisateurs discursifs dominants. Afin d'éviter toute confusion, nous avons distingué les genres dominants et les sous-genres. Les premiers ont été nommés « séquences discursives » et sont au nombre de quatre :

- les séquences descriptives, portant le plus souvent, mais pas obligatoirement sur l'image ;

- les séquences commentatives, portant sur l'image ou sur autre chose ; 
- les séquences narratives, portant sur l'expérience personnelle de l'enfant ou de l'adulte ;

- les séquences métacommunicationnelles, portant sur le déroulement de l'interaction.

Les seconds sont plus nombreux, mais n'apparaissent pas tous à la même fréquence dans notre corpus. Par conséquent, pour cette étude, nous avons retenu les quatre principaux sous-genres discursifs qui sont :

- le descriptif dont la définition est identique à celle des séquences descriptives ;

- le dénominatif qui correspond à une demande de dénomination ou une dénomination qu'elle soit produite suite à une demande ou non ;

- le narratif qui est un énoncé construisant un récit, mais n'entrant dans aucune autre catégorie d'énoncé participant également à la construction du récit (descriptif, informatif, etc.) ;

- le fictif qui est énoncé qu'un interlocuteur produit à l'aide de son imagination. Nous y incluons également toutes les hypothèses que les interlocuteurs émettent sur l'état, l'émotion ou l'action futures ou passées des personnages ou des objets se trouvant sur l'image. Etant donné que toutes ces situations relèvent de l'imaginaire plutôt que de la description, nous les avons classées dans les énoncés fictifs.

Nous présenterons, dans un premier temps, les résultats des séquences discursives qui constituent les organisateurs dominants dans les interactions de notre corpus, et dans un second temps, les résultats des sous-genres discursifs.

\subsubsection{Les séquences discursives}

L'analyse des séquences discursives nous a permis d'avoir une vue générale de notre corpus quant à la question du genre. Avant d'en présenter les résultats, nous allons illustrer chaque séquence discursive par un exemple.

- $\quad$ Exemple séquence descriptive (Yusuf, $\mathrm{T} 2^{\mathrm{i}}-\mathrm{S} 5^{\mathrm{ii}}-\mathrm{F} 4 ; 8^{\mathrm{iii}}$ ) :

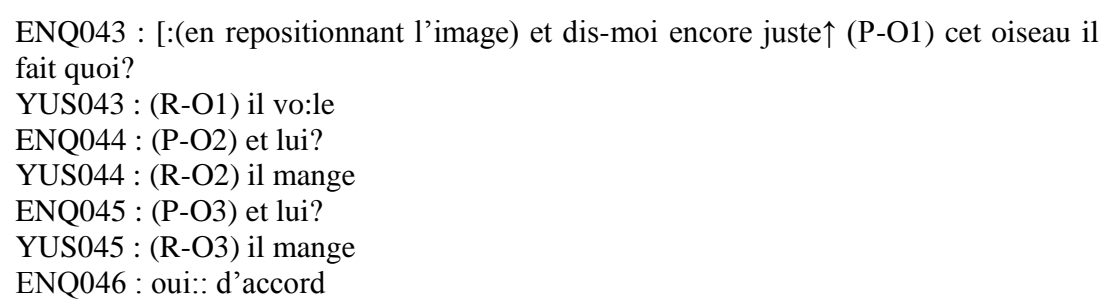

L'exemple ci-dessus, extrait de la dernière séance réalisée avec Yusuf (Type 2) à la fin de la moyenne section, est une séquence descriptive portant sur le thème des actions réalisées par les oiseaux sur l'image (ENQ043-ENQ046).

- $\quad$ Exemple de séquence commentative (Isa, T2-S3-F4;4) :

ENQ052 : il joue à la balle oui: $\uparrow+$ est-ce qu'il y a une autre balle sur l'image?

ISA053 : (P-BF)

ENQ053 : très bien $\uparrow++$ est-ce que quelqu'un joue avec cette balle?

ISA054 : (P-GC et regarde l'enquêteur)

ENQ054 : oui: (P-GC) le garçon il joue avec la balle qui est dans sa main (P-BF) mais avec cette balle personne ne joue ++ $(\mathrm{P}-\mathrm{BF})$ est-ce que toi tu aimes bien jouer avec cette balle?

ISA055: (hochement de tête négatif)

ENQ055: t'aimes pas?

ISA056: (hochement de tête négatif)

ENQ056 : qu'est-ce qu'on peut jouer avec (P-BF) cette balle?

ISA057: ++ (regarde l'enquêteur)

ENQ057 : on peut jouer au foot ++ n'est-ce pas? (P-BF) avec cette balle $\downarrow++$ on joue

au foot ISA058 : (regarde l'enquêteur)

ENQ058 : t'aimes bien jouer au foot? 
ISA059: (hochement de tête négatif)

ENQ059 : non? + d'accord

ISA060 : (regarde l'enquêteur)

ENQ060 : (en dessinant le contour de la cour) je pense qu'aucun des enfants n'aime jouer au foot $(\mathrm{P}-\mathrm{BF})$ parce que personne ne joue avec cette balle + personne n'aime jouer au foot ici

ISA061: (acquiesce en regardant l'enquêteur)

ENQ061 : ensuite (P-GME) ce garçon il fait quoi?

Dans l'extrait ci-dessus, nous pouvons observer un passage d'une séquence descriptive autour du thème du ballon de football (ENQ052-ENQ054) à une séquence commentative introduite par l'enquêteur au tour de parole ENQ054 avec la première phrase surlignée. L'enquêteur se sert d'un élément de l'image, le ballon de football pour interrompre la description et introduire une discussion autour de cet objet. Le retour à la description s'opère au tour de parole ENQ061.

- $\quad$ Exemple de séquence narrative(Ismail, T1-S4-T4;9) :

ISM104 : eskiden şey vardı: babamın gözlügü: dans le temps il y avait truc les lunettes de mon papa

ENQ105 : evet: oui

ISM105 : sonra a- annem bâton aldıydı yerden attıdı köpek ölsün diye $\uparrow+$ sonra gözlüğü kırdı bâton da $\uparrow$ après ma maman elle avait pris un bâton par terre et elle l'avait lancé pour

ENQ106: hm que le chien meure + après le bâton a cassé ses lunettes

ISM106 : sonra eve gittik yeni gözlük aldık $\uparrow$ après on est rentré à la maison on a pris de nouvelles lunettes

ENQ107 : hm: kötü olmuş + gözlüğ̈̈ mü attı köpeğe yakalasın diye anlamadım? hm c'est dommage + elle a lancé les lunettes au chien pour qu'il attrape? je n'ai pas compris

ISM107 : hayır eşya att1 non elle a lancé un objet

ENQ108 : hm eşya attı: ++ başka:? bi şey kaldı mı söylemediğimiz? hm elle a lancé un objet ++ quoi d'autre? est-ce qu'il reste quelque chose qu'on n'a pas dit?

L'exemple ci-dessus est une séquence narrative portant sur une expérience personnelle d'Ismail. Nous pouvons ajouter que c'est une séquence co-construite en raison des interventions de l'enquêteur afin d'aider l'enfant.

- $\quad$ Exemple de séquence métacommunicationnelle (Yusuf, T2-S2-F3;6) :

ENQ058 : (bruit extérieur) +++ (à Yusuf) y a quelqu'un?

YUS058 : (hausse les épaules en regardant dans la direction du bruit)

ENQ059: attends

YUS059: (salue la caméra en souriant)

ENQ060 : (en manipulant la caméra) y a qui là-bas?

YUS060 : (regarde l'enqueteur en souriant) Yusuf

ENQ061 : Yusu:f ++ (en retournant l'écran) voilà + y a toujours Yusuf mais c'est moi qui peut voir

YUS061 : ++ (en ouvrant la main) moi aussi $\uparrow[$ :(en ouvrant les deux mains) moi aussi

ENQ062 : (s'assoit à côté de Yusuf) là moi non plus je vois pas

YUS062: hm

ENQ063 : alors qu'est-ce qu'on disait Yusuf? (range l'affiche publicitaire) ça on enlève $\uparrow$

YUS063 : (se lève et essaye de retourner l'écran vers lui)

ENQ064 : tu veux tu veux te voir? (retourne l'écran vers Yusuf)

YUS064 : (content, Yusuf s'assoit en souriant)

ENQ065 : alors on laisse comme ça? 
YUS065 : (en regardant l'écran de la caméra) ouais

ENQ066 : mais tu me racontes ce que tu vois sur l'image

YUS066 : (regarde l'enquêteur puis répond en regardant l'écran de la caméra) ouais ENQ067 : d'accord?

YUS067 : (hochement de tête approbatif) (soulève l'image devant la caméra pour la voir à l'écran)

ENQ068 : non c'est bon c'est pas la peine que tu montres l'image moi je sais ce qu'il y a dessus

YUS068: (touche l'écran de la caméra)

ENQ069 : (intervient) non touche pas + parce qu'après elle va tomber (s'assoit à côté de Yusuf) alo:rs qu'est-ce qu'on disait Yusuf? ++ alors on disait que (P-EV3) cette fille elle regarde $\uparrow$

YUS069 : (en regardant la caméra) ouais

Le dernier extrait ci-dessus est une séquence métacommunicationnelle qui débute suite à un bruit extérieur qui vient interrompre le récit d'un évènement personnel que Yusuf était en train de raconter. Ensuite, se produit un incident technique et l'enquêteur essaye de remettre en route l'enregistrement vidéo. Pendant ce temps, Yusuf commence aussi à s'intéresser à la caméra. C'est seulement au tour de parole ENQ069 que l'enquêteur introduit une nouvelle séquence descriptive.

Le tableau ci-dessous présente la distribution de ces organisateurs dominants dans les interactions de notre corpus. Nous avons fait le choix de ne pas présenter dans le tableau l'évolution des pourcentages des différentes séquences discursives au fil des séances, car, d'un point de vue développemental, les résultats n'indiquent pas des différences très significatives chez les deux types d'enfant quelle que soit la langue de l'interaction.

Tableau 3: La distribution (en moyenne) des séquences discursives dans les interactions en turc et en français avec les enfants Type 1 et Type 2.

\begin{tabular}{|c|c|c|}
\hline Enfants Type 1 & Turc & Français \\
\hline Descriptives & $49,7 \%$ & $59,4 \%$ \\
\hline Commentatives & $32,9 \%$ & $25,3 \%$ \\
\hline Narratives & $7,7 \%$ & $1,5 \%$ \\
\hline Metacommunicationnelles & $9,7 \%$ & $13,8 \%$ \\
\hline
\end{tabular}

\begin{tabular}{|c|c|c|}
\hline Enfants Type 2 & Turc & Français \\
\hline Descriptives & $58,7 \%$ & $57,5 \%$ \\
\hline Commentatives & $28,5 \%$ & $26,7 \%$ \\
\hline Narratives & $2,2 \%$ & $2,6 \%$ \\
\hline Metacommunicationnelles & $10,6 \%$ & $13,2 \%$ \\
\hline
\end{tabular}

D'après les résultats ci-dessus, la première observation que nous pouvons faire est que la moyenne de toutes les séances pour chaque séquence discursive est très proche chez les deux types d'enfant dans les interactions en français. La seule différence significative se présente au niveau des séquences narratives qui sont plus nombreuses dans les interactions avec les enfants Type 2 par rapport à celles des interactions Type 1. Nous pouvons à nouveau mettre en lien ce résultat avec la pratique plus précoce du français à la maison, comme dans le cas de la proposition de thème (cf. 3.1).

À l'exception de ce point, c'est au niveau des interactions en turc que les résultats se diversifient davantage : les enfants Type 1 produisent plus de séquences narratives et commentativesalors que les enfants Type 2 produisent plus de séquences descriptives.

La distribution des séquences discursives indique donc de manière générale les principaux genres qui apparaissent dans les interactions sans chercher à savoir si l'usage de tel ou tel genre est fait par l'adulte ou par l'enfant. Afin de comprendre ces dynamiques, nous avons analysé les sous-genres discursifs séparément chez l'adulte et chacun des enfants dans chaque interaction. 


\subsubsection{Les sous-genres discursifs}

L'analyse des sous-genres discursifs nous a permis d'observer la nature de chaque énoncé produit par l'adulte et par les enfants. Avant d'en présenter les résultats, nous allons également illustrer par un exemple tiré de notre corpus, chacun des quatre principaux sous-genres discursifs que nous avons retenus pour cette étude.

- $\quad$ Exemple d'énoncé descriptif (Yusuf, T2-S5-F4;8) :

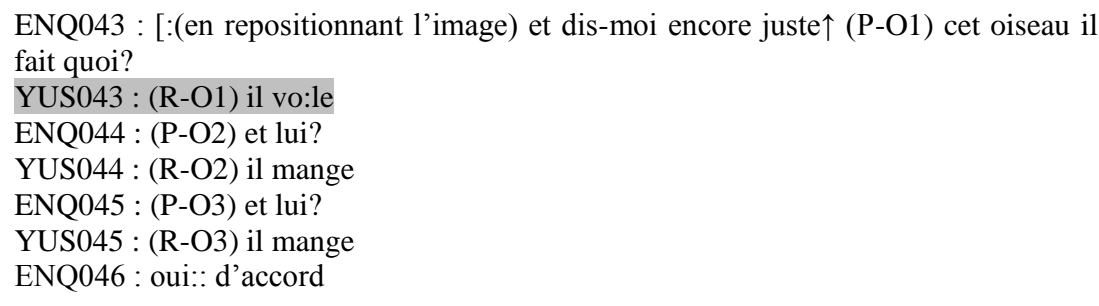

Comme l'énoncé surligné (YUS043) dans l'extrait ci-dessus, que nous avons présenté plus haut afin d'illustrer la séquence descriptive, un énoncé descriptif représente chacun des énoncés produit afin de décrire un personnage, un objet ou une situation.

- $\quad$ Exemple d'énoncé dénominatif (Asli, T1-S5-F4;6) :

ENQ113 : (P-MAIT2) et elle c'est qui?

ASL113 : maitresse (regarde l'enquêteur)

Dans l'exemple ci-dessus, nous pouvons observer deux énoncés dénominatifs : le premier (ENQ113) est une demande de dénomination adressée par l'adulte et le second (ASL113) est une dénomination fournie en guise de réponse à cette demande.

- $\quad$ Exemple d'énoncé narratif (Ismail, T1-S4-T4;9) :

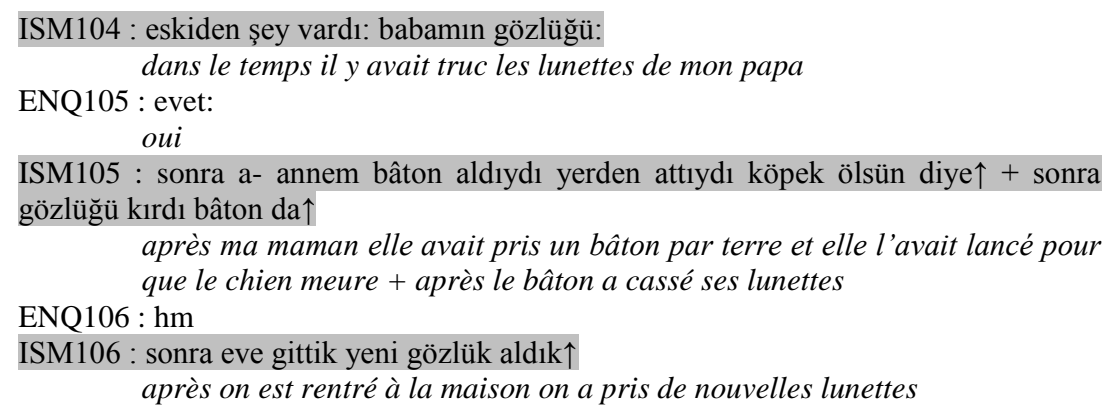

Dans cet extrait, dont nous avons présenté plus haut la version complète pour illustrer la séquence narrative, chacun des énoncés surlignés sont des énoncés narratifs produits par Ismail.

- $\quad$ Exemple d'énoncé fictif(Ismail, T1-S4-T4;9) :

ENQ092 : şunu soruyum sana + mhm: bu resimdeki çocukların sayısı sizin sınıftakilerden daha $\mathrm{m} ı$ az daha $\mathrm{m} ı$ çok?

je vais te poser une question + mhm est-ce que les enfants sur cette image sont plus nombreux ou moins nombreux que ceux de votre classe?

ISM092 : daha az moins nombreux

ENQ093 : daha az dimi? moins nombreux n'est-ce pas? 


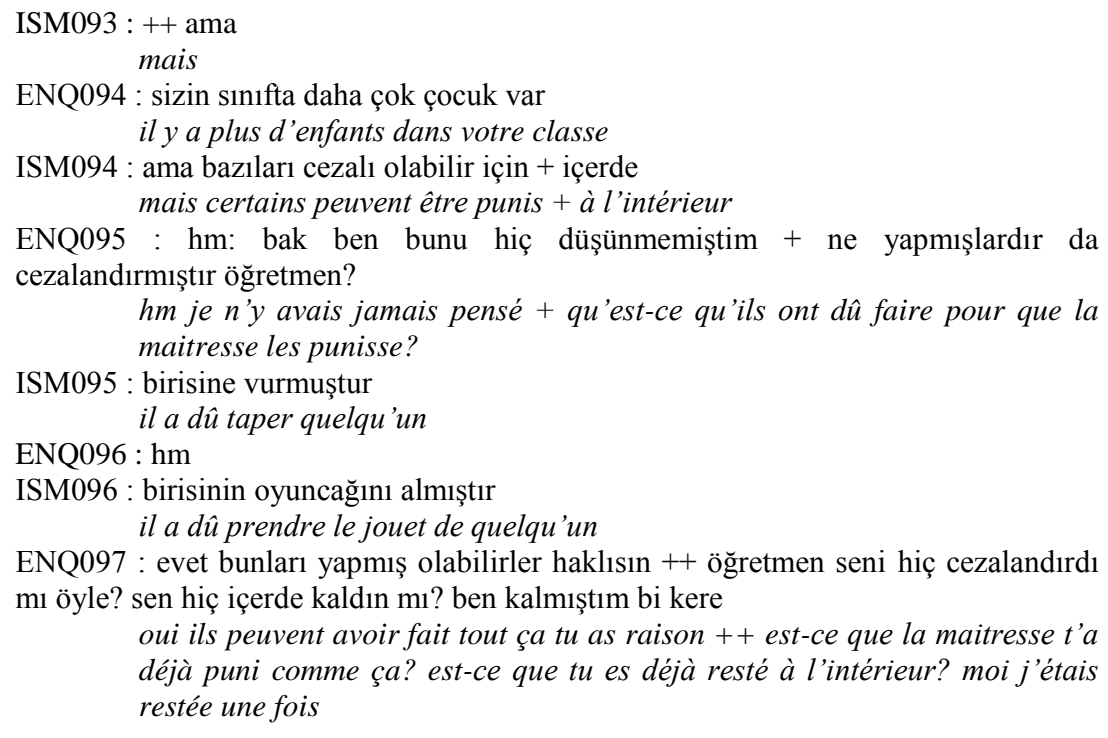

Au début de l'extrait ci-dessus, l'enquêteur demande à Ismail d'estimer le nombre d'enfants en train de jouer dans la cour de récréation sur l'image. Ismail juge qu'il y a moins d'enfants sur l'image par rapport à l'effectif de leur classe mais imagine qu'il peut également y avoir des enfants punis à l'intérieur de l'école, qui ne sont donc pas visibles sur l'image (ISM094). Ensuite, suite à la demande de l'enquêteur, il fait des suppositions sur la raison de la punition (ISM095, ISM096). Toutes ces trois interventions d'Ismail relèvent donc du fictif, étant donné qu'il ne décrit pas un évènement visible sur l'image.

Nous avons noté la distribution de ces sous-genres discursifs entre les interlocuteurs et son évolution entre la première et la dernière séance enregistrées. Le tableau ci-dessous présente les résultats des principaux sous-genres discursifs qui sont le descriptif, le dénominatif, le narratif et le fictif. La lettre « $\mathrm{A}$ » représente «l'adulte » et la lettre « $\mathrm{E} »$ représente « les enfants »:

Tableau 4: La distribution (en pourcentage) et l'évolution des principaux sous-genres discursifs chez l'adulte et les deux types d'enfant dans les interactions en turc et en français.

\begin{tabular}{|l|c|c|c|c|c|}
\cline { 3 - 6 } \multicolumn{2}{c|}{} & \multicolumn{4}{c|}{ Enfants Type 1 } \\
\cline { 2 - 6 } \multicolumn{2}{c|}{} & \multicolumn{2}{c|}{ Turc } & \multicolumn{2}{c|}{ Français } \\
\cline { 2 - 6 } \multicolumn{1}{c|}{} & $\mathrm{S} 1$ & $\mathrm{~S} 5$ & $\mathrm{~S} 1$ & $\mathrm{~S} 5$ \\
\hline \multirow{2}{*}{ Descriptif } & $\mathrm{A}$ & $28,9 \%$ & $59,8 \%$ & $16,5 \%$ & $61,8 \%$ \\
\cline { 2 - 6 } & $\mathrm{E}$ & $9,4 \%$ & $35 \%$ & $8,5 \%$ & $26,7 \%$ \\
\hline \multirow{2}{*}{ Dénominatif } & $\mathrm{A}$ & $17,2 \%$ & $2,1 \%$ & $23,1 \%$ & $4,5 \%$ \\
\cline { 2 - 6 } & $\mathrm{E}$ & $10,1 \%$ & $5 \%$ & $26,8 \%$ & $6,2 \%$ \\
\hline \multirow{2}{*}{ Narratif } & $\mathrm{A}$ & $0,6 \%$ & $1,4 \%$ & $0 \%$ & $0 \%$ \\
\cline { 2 - 6 } & $\mathrm{E}$ & $2,3 \%$ & $4,6 \%$ & $0 \%$ & $0 \%$ \\
\hline \multirow{2}{*}{ Fictif } & $\mathrm{A}$ & $1,1 \%$ & $4 \%$ & $0 \%$ & $4 \%$ \\
\cline { 2 - 6 } & $\mathrm{E}$ & $0,3 \%$ & $12,1 \%$ & $0 \%$ & $5 \%$ \\
\hline
\end{tabular}

\begin{tabular}{|c|c|c|c|}
\hline \multicolumn{3}{|c|}{ Enfants Type 2 } \\
\hline \multicolumn{2}{|c|}{ Turc } & \multicolumn{2}{c|}{ Français } \\
\hline $\mathrm{S} 1$ & $\mathrm{~S} 5$ & $\mathrm{~S} 1$ & $\mathrm{~S} 5$ \\
\hline $38,8 \%$ & $75 \%$ & $23,9 \%$ & $54,8 \%$ \\
\hline $24,4 \%$ & $28,1 \%$ & $24 \%$ & $42,7 \%$ \\
\hline $19,1 \%$ & $2,7 \%$ & $25,4 \%$ & $2,2 \%$ \\
\hline $18,5 \%$ & $6,8 \%$ & $40,7 \%$ & $3,8 \%$ \\
\hline $0 \%$ & $0,1 \%$ & $0 \%$ & $0 \%$ \\
\hline $0 \%$ & $2,1 \%$ & $0 \%$ & $0 \%$ \\
\hline $4 \%$ & $6,1 \%$ & $2,9 \%$ & $8 \%$ \\
\hline $1,8 \%$ & $4,7 \%$ & $1,2 \%$ & $14,7 \%$ \\
\hline
\end{tabular}


D'après les résultats ci-dessus, notre première observation porte sur l'effet du support imagé dans les interactions. En effet, quelle que soit la séance, la langue de l'interaction, le type d'enfant ou l'interlocuteur (adulte ou enfant), ce sont les énoncés descriptifs qui dominent. Cette domination est donc probablement due à la nature du matériel proposé et la tâche demandée aux enfants. De plus, non seulement les énoncés descriptifs dominent, mais ils augmentent aussi au fil des séances et de manière importante aussi bien chez l'adulte que les chez les enfants. Cette augmentation est beaucoup plus marquée chez les enfants Type 1 qui décrivent très peu dans les deux langues aux premières séances aussi bien par rapport aux enfants Type 2 que par rapport à l'adulte. À l'inverse des énoncés descriptifs, les énoncés dénominatifs baissent de manière considérable au fil des séances chez les deux types d'enfants, quelle que soit la langue de l'interaction.Cependant, lorsque nous observons de plus près, nous remarquons que les pourcentages d'énoncé dénominatif sont plus élevés en français chez les deux types d'enfants à 3 ans, à savoir 26,8\% pour les enfants Type 1 et 40,7\% pour les enfants Type 2 . L'adulte produit également le plus d'énoncés dénominatifs lors de la première séance en français quel que soit le type d'enfant. Mais nous pouvons aussi noter que le pourcentage de dénominatifs produits par les enfants Type 2 lors de cette première séance en français $(40,7 \%)$ est proportionnellement plus élevé $(+13,9 \%)$ par rapport au pourcentage de dénominatifs produits par les enfants Type 1 à la même séance. Si nous considérons que les enfants produisent davantage de dénominations et que l'adulte formule en général des demandes de dénomination, ce résultat indique qu'à 3 ans, en français, les enfants Type 2 produisent plus de dénominations de manière autonome par rapport aux enfants Type 1. Autrement dit, au début de la scolarisation, dans les interactions en français toutes les dénominations produites par les enfants Type 2 ne sont pas des réponses aux demandes de dénominations formulées par l'adulte alors que les enfants Type 1 ne font que répondre à ces demandes à cet âge. Nous pouvons également noter que, toujours à la première séance, mais cette fois dans les interactions en turc, les énoncés dénominatifs sont plus élevés (+ $8,4 \%$ ) chez les enfants Type 2. Cela montre qu'en turc, ces derniers ont davantage recours aux énoncés dénominatifs par rapport aux enfants Type 1.

Lorsque nous observons les énoncés narratifs, nous constatons que leur pourcentage augmente pour les deux types d'enfant dans les interactions en turc.Cependant, cette augmentation n'est pas de même nature pour les deux types d'enfants, car les enfants Type 1 produisent déjà des énoncés narratifs et davantage que l'adulte à 3 ans alors que les enfants Type 2 partent d'un taux d'énoncé narratif nul à 3 ans pour arriver à un taux de $2,1 \%$ (contre $4,6 \%$ chez les enfants Type 1) à 5 ans. L'augmentation du pourcentage d'énoncé narratif en turc n'est donc pas aussi importante chez les enfants Type 2 par rapport à celle des enfants Type 1.Dans les interactions en français, les résultats sont également intéressants. Chez les deux types d'enfant, le pourcentage des énoncés narratifs est nulle aussi bien à la première qu'à la dernière séance. Cela veut dire que l'adulte et les deux types d'enfant produisent des énoncés narratifs en français plutôt lors des séances intermédiaires. La moyenne des séquences discursives (cf. 3.2.1.) nous a également montré que les séquences narratives en français sont plus importantes chez les enfants Type 2 $(+1,1 \%)$ par rapport aux enfants Type 1 .

Quant aux énoncés fictifs, nous observons, à nouveau, une augmentation au fil des séances quel que soit le type d'enfant et la langue de l'interaction.Cependant, cette augmentation est plus marquée en turc chez les enfants Type $1(+11,8 \%$ contre $+2,9 \%$ chez les enfants Type 2$)$ alors qu'elle est plus marquée en français chez les enfants Type $2(+13,5 \%$ contre $+2,9 \%$ chez les enfants Type 1$)$.Autrement dit, à 5 ans, les enfants Type 1 produisent davantage d'énoncé fictif en turc alors que les enfants Type 2 en produisent davantage en français. Il faut également noter qu'à 3 ans, les enfants Type 1 partent d'un pourcentage nul d'énoncé fictif en français (contrairement aux enfants Type 2, qui eux, en produisaient déjà à 3 ans), pour arriver, à 5 ans, à un taux d'énoncé fictif de $5 \%$, ce qui est une bonne évolution pour ces enfants qui ne pratiquent pas le français à la maison au même degré que les enfants Type 2. Nous nous demandons également si l'absence d'énoncé fictif chez les enfants Type 1 à 3 ans n'est pas justement dû au fait que l'adulte n'en produise pas non plus à la première séance, alors qu'elle en produit avec les enfants Type 2.

En résumé, à l'exception des énoncés dénominatifs dont la production baisse, celle des énoncés descriptifs, narratifs et fictifs augmente au fil des séances aussi bien chez l'adulte que chez les enfants. 
Après avoir observé la nature des énoncés dans le cadre de la question du genre, nous observerons maintenant la fonction des énoncés dans les interactions.

\subsection{Les modalités d'énoncé}

Nous entendons par modalité d'énoncé les différentes modalités d'intervention de l'adulte et des enfants. Le tableau ci-dessous présente les résultats des trois modalités d'énoncé parmi les sept grandes catégories de modalité d'énoncé que nous avons relevées dans notre corpus. La catégorie des questions contient toutes les demandes de l'adulte ou de l'enfant sauf les ordres qui constituent une catégorie à part entière dont nous ne présenterons pas les résultats dans ce travail. Les différents types de question sont : les questions classiques, les assertions sollicitantes qui requièrent une réponse de la part de l'interlocuteur, les ébauches, les relances, les demandes de confirmation, les demandes de clarification, les demandes de répétition et enfin, les demandes de changement de langue qui sont toujours adressées par l'adulte lorsque l'enfant répond dans la langue qui n'est pas celle de l'interaction. Quant aux assertions, elles correspondent à toutes les interventions qui ne sont pas des réponses aux questions. Etant donné que les assertions sont des énoncés produites sans la sollicitation de l'interlocuteur, nous les considérons comme une indication du degré d'autonomie de l'enfant dans une interaction. Les assertions correspondent, en général, à des propositions de thème (cf. 3.1) ou à des énoncés constituant une séquence narrative où les interlocuteurs sont plus autonomes par rapport aux autres séquences discursives (cf. 3.2.1.).

Tableau 5: La distribution (en pourcentage) et l'évolution des principales modalités d'énoncés chez l'adulte et les deux types d'enfant dans les interactions en turc et en français.

\begin{tabular}{|l|c|c|c|c|c|}
\cline { 3 - 6 } \multicolumn{1}{c|}{} & \multicolumn{4}{c|}{ Enfants Type 1 } \\
\cline { 2 - 6 } \multicolumn{2}{c|}{} & \multicolumn{2}{c|}{ Turc } & \multicolumn{2}{c|}{ Français } \\
\cline { 2 - 6 } \multicolumn{1}{c|}{} & S1 & S5 & S1 & S5 \\
\hline \multirow{2}{*}{$\begin{array}{l}\text { Question } \\
\text { Réponse }\end{array}$} & $\mathrm{A}$ & $40,2 \%$ & $31,3 \%$ & $42,7 \%$ & $34,4 \%$ \\
\cline { 2 - 6 } & $\mathrm{E}$ & $48,9 \%$ & $53,7 \%$ & $38,7 \%$ & $41,1 \%$ \\
\hline \multirow{3}{*}{ Assertion } & $\mathrm{A}$ & $20,2 \%$ & $20,9 \%$ & $24,7 \%$ & $15 \%$ \\
\cline { 2 - 6 } & $\mathrm{E}$ & $15,1 \%$ & $24,9 \%$ & $19,4 \%$ & $15,9 \%$ \\
\hline
\end{tabular}

\begin{tabular}{|c|c|c|c|}
\hline \multicolumn{3}{|c|}{ Enfants Type 2 } \\
\hline \multicolumn{2}{|c|}{ Turc } & \multicolumn{2}{c|}{ Français } \\
\hline $\mathrm{S} 1$ & $\mathrm{~S} 5$ & $\mathrm{~S} 1$ & $\mathrm{~S} 5$ \\
\hline $41,3 \%$ & $44,4 \%$ & $37,4 \%$ & $44,5 \%$ \\
\hline $60 \%$ & $62,5 \%$ & $29,8 \%$ & $49,8 \%$ \\
\hline $18,5 \%$ & $18,5 \%$ & $9,5 \%$ & $17,6 \%$ \\
\hline $15,5 \%$ & $6,7 \%$ & $14,3 \%$ & $37,3 \%$ \\
\hline
\end{tabular}

Toutes les interactions de notre corpus sont fondées sur les échanges question/réponse. En général, c'est l'adulte qui pose les questions et l'enfant qui donne les réponses. Or, les résultats ci-dessus nous montrent que le comportement de l'adulte n'est pas le même en fonction du type d'enfant car ce dernier pose plus de questions aux enfants Type 2 que ce soit en turc ou en français.

Sur le plan développemental, l'adulte questionne de moins en moins les enfants Type 1 au fil des séances et ce dans les deux langues. Les séances intermédiaires dont les taux ne figurent pas sur le tableau indiquent également une baisse régulière des questions de l'adulte au fil des séances. Quant aux interactions avec les enfants Type 2, nous constatons que l'adulte pose de plus en plus de questions au fil des séances, à l'inverse de son comportement avec les enfants Type 1.

En ce qui concerne les assertions, en turc, elles n'évoluent presque pas chez l'adulte entre la première et la dernière séance. Cependant, en français, le taux d'assertion de l'adulte baisse dans les interactions avec les enfants Type 1 (- 9,7\%) alors qu'il augmente dans les interactions avec les enfants Type $2(+8,1 \%)$. Quant aux énoncés des enfants, en turc, les enfants Type 1 produisent plus d'assertions à 5 ans qu'à 3 ans $(+9,8 \%)$ alors qu'en français, ils en produisent moins $(-3,5 \%)$. En ce qui concerne les enfants Type 2 , 
nous observons le contraire : leur pourcentage d'assertion baisse en turc (- 8,8 \%) alors qu'il augmente considérablement en français $(+23 \%)$ entre 3 et 5 ans. La comparaison des deux types d'enfant indique quelques différences. Or, d'un point de vue interactionnel, la lecture de ces résultats ne sera complète qu'après une comparaison avec ceux de l'adulte. Dans les interactions avec les enfants Type 1, nous observons que malgré une légère domination de l'adulte à la première séance, à 5 ans, les pourcentages d'assertion de l'adulte sont très proches de ceux des enfants, que ce soit en turc ou en français. Cela montre que les enfants Type 1 ont le même degré d'autonomie que l'adulte dans les deux langues. Cependant, nous ne pouvons faire la même observation en ce qui concerne les interactions avec les enfants Type 2. En effet, dans les interactions en turc, c'est malgré la stabilité du taux d'assertion de l'adulte à la première et à la dernière séance $(18,5 \%)$ que celui des enfants Type 2 diminue de manière considérable et passe de $15,5 \%$ à 3 ans à $6,7 \%$ à 5 ans $(-8,8 \%)$. Cette diminution peut être le résultat d'une perte d'autonomie en turc, langue qu'ils pratiquent moins au profit du français depuis leur scolarisation. À l'inverse, dans les interactions en français, nous observons que ce sont les taux d'assertion des enfants Type 2 qui sont supérieurs à ceux de l'adulte aussi bien à la première qu'à la dernière séance. De plus, contrairement à la diminution dans les interactions en turc, leurs assertions augmentent de manière considérable $(+23 \%)$ entre 3 et 5 ans alors que les assertions de l'adulte n'augmentent seulement de $8,1 \%$ entre la première et la dernière séance. Les enfants Type 2 sont donc beaucoup plus autonomes en français.

Par conséquent, ces résultats indiquentune autonomie plus importante en turc pour les enfants Type 1 et en français pour les enfants Type 2, ce qui semble cohérent par rapport à leurs pratiques langagières familiales.

Notre dernier volet d'analyse porte sur la diversité lexicale dans les productions des enfants.

\subsection{La diversité lexicale}

Notre dernier volet d'analyse n'est pas interactionnel dans le sens où nous n'avons pas mesuré la diversité lexicale de l'adulte et ses effets sur celle des enfants et inversement, ce qui fera l'objet d'une prochaine étude. Dans le tableau ci-dessous, nous présentons uniquement les résultats de diversité lexicale dans les productions orales des deux types d'enfant en turc et en français et leur évolution entre la première (3 ans) et la dernière séance (5 ans). Le calcul a été réalisé avec l'indice de Guiraud (Guiraud, 1954) qui consiste à diviser le nombre de mots différents $(\mathrm{V})$ produits par l'enfant dans une interaction par la racine carré du nombre total de mots produits $(\mathrm{N})$. La formule de Guiraud est donc la suivante : $\mathrm{G}=\mathrm{V} / \sqrt{ } \mathrm{N}$.

Tableau 6: L'évolution (en indice de Guiraud) de la diversité lexicale des enfants Type 1 et Type 2 en turc et en français.

\begin{tabular}{|c|c|c|c|}
\hline \multicolumn{4}{|c|}{ Enfants Type 1 } \\
\hline \multicolumn{2}{|c|}{ Turc } & \multicolumn{2}{c|}{ Français } \\
\hline S1 & S5 & S1 & S5 \\
\hline 4,2 & 5,4 & 0,8 & 5,3 \\
\hline
\end{tabular}

\begin{tabular}{|c|c|c|c|}
\hline \multicolumn{4}{|c|}{ Enfants Type 2 } \\
\hline \multicolumn{2}{|c|}{ Turc } & \multicolumn{2}{c|}{ Français } \\
\hline S1 & S5 & S1 & S5 \\
\hline 3,9 & 4,6 & 2,4 & 5,6 \\
\hline
\end{tabular}

Avant d'observer les résultats en détail, tout d'abord, nous pouvons noter que la diversité lexicale des deux types d'enfant augmente entre 3 et 5 ans, et ce dans les deux langues. Ensuite, nous remarquons qu'à 3 ans, les deux types d'enfants ont une diversité lexicale plus élevée en turc, avec un taux plus important pour les enfants Type 1 (4,2 contre 3,9 chez les enfants Type 2). Autrement dit, tous les enfants d'origine turque que nous avons observés et d'autant plus les enfants Type 1 pratiquant davantage le turc à la 
maison, ont un lexique plus riche en turc au début de la scolarisation, ce qui n'est pas un résultat inattendu étant donné que la pratique du français ne commence à être renforcée qu'à partir de 3 ans avec la scolarisation. En revanche, en français, les enfants Type 1 ont une diversité lexicale très faible par rapport à celle des enfants Type 2, ce qui est probablement dû à la pratique plus précoce du français.

Dans leurs productions en turc, nous observons que les enfants Type 1 préservent un taux de diversité lexicale supérieur à celui des enfants Type 2 même au bout de deux années de scolarisation en français. De plus, leur diversité lexicale augmente également en français, et ce de manière considérable pour atteindre un taux de diversité lexicale très proche de celui des enfants Type 2 à 5 ans. Par conséquent, les enfants Type 1 atteignent à la fois, en turc, une meilleure diversité lexicale grâce à la pratique du turc à la maison et, en français, presque le même taux de diversité lexicale que les enfants Type 2 grâce à la pratique du français à l'école.

L'équilibre de la diversité lexicale dans les deux langues chez les enfants Type 1 à 5 ans est également intéressant à noter. En effet, ces derniers ont presque le même taux de diversité lexicale dans leurs deux langues, à savoir 5,4 en turc et 5,3 en français. En revanche, nous ne retrouvons pas cet équilibre entre les deux langues des enfants Type 2 car leur diversité lexicale est nettement plus élevée en français à 5 ans.

Ces résultats montrent donc qu'au bout de deux années de scolarisation, les enfants turcs atteignent le même niveau de diversité lexicale en français quelles que soient leurs pratiques langagières familiales.

Les différences entre les deux types d'enfant se présentent plutôt en turc. À 5 ans, nous pouvons dire que les enfants Type 1 n'ont pas de langue dominante au niveau de la diversité lexicale alors que les enfants Type 2 semblent plutôt dominants en français, avec l'effet de l'école qui s'ajoute à celui des pratiques langagières familiales.

\section{Conclusion}

Dans le cadre de cette étude, nous nous sommes interrogés sur les effets des pratiques langagières familiales dans le développement du bilinguisme émergent des enfants d'origine turque issus de familles immigrées scolarisés en maternelle. Notre analyse des données et les résultats qui en découlent nous mènent à plusieurs conclusions. Premièrement, nous avons observé qu'au bout de deux années de scolarisation, la plupart des résultats des enfants Type 1 et Type 2 présentaient peu de différences en français. Le niveau de diversité lexicale en français des deux types d'enfant à 5 ans en est, sans doute, l'exemple le plus significatif.

En revanche, d'autres résultats semblent également nous indiquer des langues dominantes pour certaines compétences chez les deux types d'enfant. Nous avons observé, par exemple, qu'en turc, les enfants Type 1 proposaient davantage de thèmes que les enfants Type 2 alors qu'en français, ce sont les enfants Type 2 qui en proposaient davantage par rapport aux enfants Type 1. Nous avons également noté qu'à 5 ans, en français, la diversité lexicale des enfants Type 2 était plus élevée que celle des enfants Type 1. Ces résultats montrent donc qu'une pratique plus fréquente du turc à la maison permet un meilleur développement de certaines compétences en turc, ce qui rend avantagés les enfants Type 1 qui pratiquent davantage le turc à la maison. De même, la pratique du français à la maison qui est ensuite renforcée par l'école à partir de 3 ans permet aux enfants Type 2 dont les parents pratiquent indifféremment les deux langues à la maison d'être dominants en français dans certains aspects de la langue.

Néanmoins, de manière générale, ces résultats montrent surtout que quelles que soient les pratiques langagières familiales des enfants d'origine turque, deux années de scolarisation en français leur permet d'égaliser certaines compétences, même si d'autres restent variées. Le résultat le plus significatif est sans doute celui de la diversité lexicale, ce qui est l'une des plus grandes sources d'inquiétude des enseignants. Nous pouvons donc proposer comme conclusion principale de cette étude que la pratique de la L1 à la maison ne nuit pas au développement de la L2, langue de l'école. 


\section{Références bibliographiques}

Akinci, M.-A., (sous presse), Le bilinguisme des enfants turcophones issus de familles immigrées. In Hélot, C. \& Erfurt, J. (éds), L'éducation bilingue en France : Politiques linguistiques, modèles et pratiques. Rennes : Presses universitaires de Rennes.

Bakhtine, M. (1984). Esthétique de la création verbale. Paris: Gallimard.

Bruner, J. (1983). Child's Talk : learning to use language. New York : Norton.

François, F. (1990). La communication inégale. Neuchâtel-Paris : Delachaux et Niestlé.

François, F. (1993). Pratiques de l'oral: dialogue, jeu et variations des figures de sens. Paris: Nathan.

François, F. \& Delamotte-Legrand, R. (2004). Enfants et récits. Mises en mots et "reste", Villeneuve d'Ascq : Presses universitaires du Septentrion.

Garcia, O. (2009). Bilingual education in the 21st century : A global perspective. Malden MA : Wiley Blackwell.

Guiraud, P. (1954). Les Caractères statistiques du vocabulaire. Paris : Presses universitaires de France.

Hamurcu Süverdem, B. (2015). Le développement du turc et du français en situation de bilinguisme précoce. Le cas d'enfants d'origine turque scolarisés en maternelle. Thèse de doctorat non publiée. Rouen : Université de Rouen.

Hélot, C. (2007). Du bilinguisme en famille au plurilinguisme à l'école. Paris : l'Harmattan.

Kerbrat-Orecchioni, C. (1990). Les interactions verbales. Tome 1 : Approche interactionnelle et structure des conversations. Paris : Armand Colin (2006).

Kern, S. \& Raffara, A. (2012). Effet du type de support imagé sur la production du récit chez l'enfant. In Delamotte, R. \& Akinci, M.-A. (dir.), Récits d'enfants. Développement, genre, contexte. Rouen : Presses Universitaires de Rouen et du Havre, pp. 97-115.

Vygotsky, L. (1934).Thought and Language. Cambridge, MA : The M.I.T. Press, (1985).

${ }^{\mathrm{i}}$ Enfant Type 2 (dont les parents parlent indifféremment les deux langues à la maison).

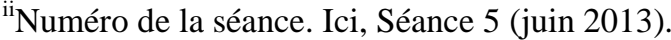

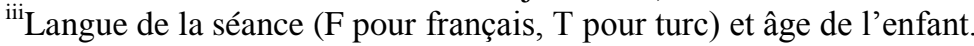

